\title{
Growth Estimates for the Numerical Range of Holomorphic Mappings and Applications
}

\author{
Filippo Bracci* Marina Levenshtein, \\ Simeon Reich ${ }^{\dagger}$ and David Shoikhet
}

July 12, 2018

AMS Mathematics Subject Classifications (2010): 47A12; 46G20; 46T25; $58 \mathrm{~B} 12$

Keywords: numerical range; growth estimates; Bloch radii; holomorphic maps; Banach spaces

\begin{abstract}
The numerical range of holomorphic mappings arises in many aspects of nonlinear analysis, finite and infinite dimensional holomorphy, and complex dynamical systems. In particular, this notion plays a crucial role in establishing exponential and product formulas for semigroups of holomorphic mappings, the study of flow invariance and range conditions, geometric function theory in finite and infinite dimensional Banach spaces, and in the study of complete and semi-complete vector fields and their applications to starlike and spirallike mappings, and to Bloch (univalence) radii for locally biholomorphic mappings.

In the present paper we establish lower and upper bounds for the numerical range of holomorphic mappings in Banach spaces. In addition, we study and discuss some geometric and quantitative analytic aspects of fixed point theory, nonlinear resolvents of holomorphic mappings, Bloch radii, as well as radii of starlikeness and spirallikeness.
\end{abstract}

\section{Introduction and preliminaries}

Let $X^{*}$ denote the dual of a complex Banach space $X$ and let $\left\langle x, x^{*}\right\rangle$ denote the duality pairing of $x \in X$ and $x^{*} \in X^{*}$. For each $x \in X$, the set $J(x)$, defined by

$$
J(x):=\left\{x^{*} \in X^{*}:\left\langle x, x^{*}\right\rangle=\|x\|^{2}=\left\|x^{*}\right\|^{2}\right\},
$$

*Partially supported by the ERC grant "HEVO - Holomorphic Evolution Equations" no. 277691.

${ }^{\dagger}$ Partially supported by the Israel Science Foundation (Grant No. 389/12), by the Fund for the Promotion of Research at the Technion and by the Technion General Research Fund 
is not empty by the Hahn-Banach theorem, and is a closed and convex subset of $X^{*}$.

Let $D$ be a domain in $X$ and let $f: D \rightarrow X$ be a mapping. We use the notation $\sup _{x \in D} \operatorname{Re}\left\langle f(x), x^{*}\right\rangle$ for the supremum of $\operatorname{Re}\left\langle f(x), x^{*}\right\rangle$ over all pairs $x \in D$ and $x^{*} \in J(x)$.

We denote by $\mathcal{B}_{R}:=\{x \in X:\|x\|<R\}$ the open ball centered at the origin of radius $R$ in the complex Banach space $X$.

Definition 1 (cf. [12] and [14]) Let $h: \overline{\mathcal{B}_{R}} \rightarrow X$ be continuous on the closure $\overline{\mathcal{B}_{R}}$ of $\mathcal{B}_{R}$. We define the set

$$
V_{\mathcal{B}_{R}}(h):=\left\{\left\langle h(x), x^{*}\right\rangle:\|x\|=R, x^{*} \in J(x)\right\}
$$

and call it the numerical range of $h$ with respect to $\mathcal{B}_{R}$.

The number $\left|V_{\mathcal{B}_{R}}(h)\right|:=\sup \left\{\left|\left\langle h(x), x^{*}\right\rangle\right|:\|x\|=R, x^{*} \in J(x)\right\}$ is called the numerical radius of $h$ with respect to $\mathcal{B}_{R}$.

We denote by $\operatorname{Hol}(\mathcal{D}, X)$ the set of all holomorphic mappings from a domain $\mathcal{D} \subset X$ into $X$.

Definition 2 (cf. [12] and [14]) Let $h \in \operatorname{Hol}\left(\mathcal{B}_{R}, X\right)$. We say that $h$ is (holomorphically) dissipative if

$$
\limsup _{s \rightarrow 1^{-}} \sup \operatorname{Re} V_{\mathcal{B}_{R}}\left(h_{s}\right) \leq 0,
$$

where $h_{s}(x):=h(s x), 0 \leq s<1$.

In view of their numerous applications, dissipative mappings which are not necessarily holomorphic constitute an important class of mappings in complex Banach spaces. In this paper we introduce the following more general notion.

Definition 3 Given $\omega, \theta \in \mathbb{R}$, a mapping $h: \mathcal{B}_{R} \rightarrow X$ is called $(\omega, \theta)$-dissipative (or just quasi-dissipative) on $\mathcal{B}_{R}$ if there exists $\varepsilon>0$ such that

$$
\operatorname{Re}\left\langle e^{i \theta} h(x), x^{*}\right\rangle \leq \omega,
$$

for all $x$ satisfying $R-\varepsilon<\|x\|<R$ and $x^{*} \in J(x)$.

For a holomorphic mapping $h$ on the unit ball $\mathcal{B}=\mathcal{B}_{1}$ the above definition means that for each $s \in(1-\varepsilon, 1)$, the closed convex hull of the numerical range of each $h_{s}$ is not the whole complex plane, or which is one and the same, $h: \mathcal{B} \rightarrow X$ is quasi-dissipative if there is $\varepsilon>0$ such that the closed convex hull of the set

$$
\Omega_{\varepsilon}(h):=\left\{\left\langle h(x), x^{*}\right\rangle: 1-\varepsilon<\|x\|<1, x^{*} \in J(x)\right\}
$$

is not the whole complex plane $\mathbb{C}$. 
Obviously, a holomorphic $(0,0)$-dissipative mapping on the unit ball is holomorphically dissipative in the sense of Definition 2 Also note that $(0, \pi)$ dissipative mappings are sometimes called holomorphically accretive [7].

One of the general problems we intend to study is the following one:

Given a quasi-dissipative mapping $h$ on the open unit ball $\mathcal{B}$, find $r \in(0,1)$ (if it exists) such that $h$ is dissipative on the ball $\mathcal{B}_{r}$.

Since every holomorphic mapping $h$ on a domain $\mathcal{D}$ is locally Lipschitzian, it follows that the Cauchy problem

$$
\left\{\begin{array}{l}
\frac{d x(t)}{d t}=h(x(t)) \\
x(0)=x_{0}
\end{array}\right.
$$

has a unique continuous solution $x(t)$ defined on the interval $[0, T]$, where $T$ depends on the initial value $x_{0} \in \mathcal{D}$.

The mapping $-h$ is said to be a semi-complete vector field on $\mathcal{D}$ if for each $x_{0} \in \mathcal{D}$, this solution is well defined on the right half-axis $[0, \infty)$ and the values of $x(t)$ belong to $\mathcal{D}$ for each initial data $x_{0} \in \mathcal{D}$. In this situation, $h$ generates a one-parameter semigroup of holomorphic self-mappings of $\mathcal{D}[20$.

Note that it may happen that $-h$ is not semi-complete on the whole domain of definition $\mathcal{D}$, but it is semi-complete on some open subset of $\mathcal{D}$. In this case we say that $-h$ is a locally semi-complete vector field.

It is known (see, for example, 20]) that if $h$ is holomorphic on $\mathcal{B}_{R}$, then $-h$ is semi-complete on $\mathcal{B}_{R}$ if and only if $h$ is dissipative on $\mathcal{B}_{R}$.

Moreover, it turns out that if the numerical range of $h$ (say, with respect to the open unit ball $\mathcal{B}$ of $X$ ) is not the whole complex plane, then for each $r \in(0,1)$, there is a real number $\omega=\omega(r)$ such that the perturbed mapping $\omega I-h$ is semi-complete on the ball $\mathcal{B}_{r}$. The question is how this number $\omega$ depends on $r$ and how to find the minimal value of the function $\omega(r)$ with respect to $r \in(0,1)$.

By using the exponential formula for semigroups of holomorphic mappings (see, for example, 20]) one can see that this problem is equivalent to the following one. Find a function $\omega(r)$ on the interval $(0,1)$ such that the nonlinear resolvent $(\lambda I-h)^{-1}$ is well defined on the ball $\mathcal{B}_{\rho}$ of radius $\rho=(\lambda-\omega(r)) r$ for all $\lambda \geq \omega(r)$ and maps this ball into $\mathcal{B}_{r}$. We will study this problem in more detail in the third section of our paper.

In this case it is also of interest, in analogy with the linear theory, to determine if the associated resolvent mapping $(\lambda I-h)^{-1} \circ(\lambda-\omega(r)) I$ can be extended to a sector in the complex plane with vertex at $\omega(r)$ and to estimate the angle of its opening.

For a holomorphic mapping $h: \mathcal{B} \rightarrow X$, one says that it has unit radius of boundedness if it is bounded on each subset strictly inside $\mathcal{B}$ ([8, 12]; see also $[2,3]$ ).

It follows from a result of $\mathrm{L}$. A. Harris 12 that a holomorphic mapping on $\mathcal{B}$ has unit radius of boundedness if and only if its numerical radius $\left|V_{\mathcal{D}}(h)\right|$ is bounded with respect to any convex subset $\mathcal{D}$ in $\mathcal{B}$. Moreover, it was shown in 
[14] that this is equivalent to a formally weaker condition, namely,

$$
\limsup _{r \rightarrow 1^{-}} \sup _{\|x\|=r} \operatorname{Re}\left\langle h(x), x^{*}\right\rangle<\infty .
$$

The problem of verifying whether a holomorphic mapping has unit radius of boundedness, as well as the general study of its numerical ranges, arise in many aspects of infinite dimensional holomorphy (see, for example, 8, 12]) and complex dynamical systems [1, 20. In particular, they play a crucial role in establishing exponential and product formulas for semigroups of holomorphic mappings [18, 19, the study of flow invariance and range conditions in nonlinear analysis [14, 17, and geometric function theory in finite and infinite dimensional Banach spaces [20]. They were specifically used for the class of semi-complete vector fields (or infinitesimal generators) in their applications to the study of starlike and spirallike mappings 20, and Bloch (univalence) radii 14 for locally biholomorphic mappings. Other pertinent papers include [10, [11] and [21].

Observe also that the concept of unit radius of boundedness for holomorphic mappings is a specific phenomenon in the infinite dimensional case because in a finite dimensional Banach space each holomorphic mapping on the unit ball is bounded on each subset strictly inside the ball. This is no longer true in the general case. Relevant examples can be found in [5].

\section{Lower and upper bounds for the numerical range}

Let $\mathcal{D}$ be a domain in $X, 0 \in \mathcal{D}$ and let $h: \mathcal{D} \rightarrow X$ be holomorphic. Since $h$ is locally bounded, there is a ball $\mathcal{B}_{R}$ in $\mathcal{D}$ such that

$$
N_{R}:=\sup _{\|x\|<R} \operatorname{Re}\left\langle h(x), x^{*}\right\rangle<\infty .
$$

Also, for each $r<R$, we use the quantity

$$
N_{r}:=\sup _{\|x\|=r} \operatorname{Re}\left\langle h(x), x^{*}\right\rangle .
$$

The first aim of this section is the following one.

(i) Find an explicit upper bound $\mathcal{F}(r)$ for $N_{r}$ which depends only on $h(0)$, $h^{\prime}(0)$ and $N_{R}$ such that

$$
\lim _{r \rightarrow R^{-}} \mathcal{F}(r)=N_{R}
$$

On the other hand, it might happen that for a given $R>0$, the value of $N_{R}\left(:=\sup _{\|x\|<R} \operatorname{Re}\left\langle h(x), x^{*}\right\rangle\right)$ is not bounded, while the convex hull of the numerical range of $h$ is not the whole plane. This is equivalent to the fact that for some real $\theta$,

$$
N_{R}(\theta):=\sup _{\|x\|<R} \operatorname{Re}\left\langle e^{i \theta} h(x), x^{*}\right\rangle<\infty
$$


In other words, it may happen that even if $N_{R}(\theta)$ is finite for some real $\theta$, the value $N_{R}(0)=N_{R}$ is not finite. A simple example is given in $X=\mathbb{C}$, the complex plane, by the mapping $h(x)=i x \frac{1+x}{1-x}$ with $R=1$ and $\theta=\frac{\pi}{2}$. Nevertheless, for each $r<R$, the value $N_{r}$ is finite on the smaller ball $\{x \in X$ : $\|x\| \leq r\}$.

Therefore the following problem is also relevant.

(ii) Knowing the value $N_{R}(\theta)$, find an explicit upper bound $\mathcal{F}(r, \theta)$ for $\sup _{\|x\|=r} \operatorname{Re}\left\langle h(x), x^{*}\right\rangle$, which depends only on $h(0), h^{\prime}(0), \theta$ and $N_{R}(\theta)$, and such that $\mathcal{F}(r, 0)=\mathcal{F}(r)$.

Then, by definition, this function also gives us growth estimates for the upper bound of the numerical range of $h$ with respect to $r \leq R$.

Similarly, one can consider the problem of finding growth estimates for the function

$$
M_{r}(\theta):=\sup _{\|x\|=r} \operatorname{Re}\left\langle e^{i \theta}(h(x)-h(0)), x^{*}\right\rangle
$$

by using a suitable computable function $\Psi(r, \theta)$ such that

$$
M_{r}(\theta) \leq \Psi(r, \theta) \quad \text { and } \quad \lim _{r \rightarrow R^{-}} \Psi(r, 0)=M_{R}(0),
$$

where

$$
M_{R}(\theta):=\sup _{\|x\|<R} \operatorname{Re}\left\langle e^{i \theta}(h(x)-h(0)), x^{*}\right\rangle .
$$

Let us start solving Problem (i):

Proposition 4 Let $h$ be a holomorphic mapping on $\mathcal{B}_{R}$ and assume that

$$
N_{R}=\sup _{\|x\|<R} \operatorname{Re}\left\langle h(x), x^{*}\right\rangle<\infty .
$$

Then the following estimate holds:

$$
N_{r}=\sup _{\|x\|=r} \operatorname{Re}\left\langle h(x), x^{*}\right\rangle \leq \mathcal{F}(r),
$$

where

$$
\mathcal{F}(r):=r\|h(0)\|\left(1-\frac{r^{2}}{R^{2}}\right)+\frac{r^{2}}{R+r}\left[(R-r) L+2 r \cdot \frac{N_{R}}{R^{2}}\right]
$$

and $L:=\sup _{\|u\|=1} \operatorname{Re}\left\langle h^{\prime}(0) u, u^{*}\right\rangle \leq \frac{N_{R}}{R^{2}}$.

Proof. Let $g: \Delta_{R} \rightarrow \mathbb{C}$ be a holomorphic function in $\Delta_{R}:=\{z \in \mathbb{C}:|z|<R\}$ for some $R>0$. By the Hadamard-Borel-Carathéodory inequality, for all $\zeta \in \Delta_{R}$ such that $|\zeta|=r \in(0, R)$ we have

$$
\operatorname{Re} g(\zeta) \leq \frac{R-r}{R+r} \operatorname{Re} g(0)+\frac{2 r}{R+r} \sup _{|\xi|<R} \operatorname{Re} g(\xi) .
$$


Now, let $f: \Delta_{R} \rightarrow \mathbb{C}$ be a holomorphic function and consider the holomorphic function $g: \Delta_{R} \rightarrow \mathbb{C}$ defined by

$$
g(\zeta):= \begin{cases}\frac{f(\zeta)-f(0)}{\zeta}+\frac{\overline{f(0)}}{R^{2}} \zeta, & \zeta \neq 0 \\ f^{\prime}(0) & \zeta=0 .\end{cases}
$$

Note that

$$
\begin{aligned}
\operatorname{Re} f^{\prime}(0) & =\operatorname{Re} g(0) \leq \limsup _{r \rightarrow R^{-}} \max _{|\zeta|=r} \operatorname{Re} g(\zeta) \\
& =\limsup _{r \rightarrow R^{-}} \max _{|\zeta|=r} \frac{1}{r^{2}}\left[\operatorname{Re} f(\zeta) \bar{\zeta}-\operatorname{Re}\left(f(0) \bar{\zeta}\left(1-\frac{r^{2}}{R^{2}}\right)\right)\right] \\
& \leq \frac{1}{R^{2}} \sup _{|\zeta|<R} \operatorname{Re} f(\zeta) \bar{\zeta} .
\end{aligned}
$$

Moreover, applying (6) to (7), for all $\zeta$ such that $|\zeta|=r \in(0, R)$, we obtain

$$
\begin{aligned}
\operatorname{Re}(\bar{\zeta} f(\zeta)) & \leq\left(1-\frac{r^{2}}{R^{2}}\right) \operatorname{Re}(\bar{\zeta} f(0)) \\
& +r^{2}\left[\operatorname{Re} f^{\prime}(0) \frac{R-r}{R+r}+\frac{2 r}{R^{2}(R+r)} \sup _{|\xi|<R} \operatorname{Re}(\bar{\xi} f(\xi))\right] .
\end{aligned}
$$

Let now $u \in X,\|u\|=1$. Fix $u^{*} \in J(u)$ and consider the holomorphic function $f: \Delta_{R} \rightarrow \mathbb{C}$ defined by $f(\zeta)=\left\langle h(\zeta u), u^{*}\right\rangle$. Applying (9) to this $f$ and taking into account (8), we can easily complete the proof of the proposition.

Remark 5 Note that inequality (9) is, in fact, equivalent to the HadamardBorel-Carathéodory inequality (6). Simply apply (9) to the holomorphic function $f(\zeta)=\zeta g(\zeta)$.

The solution to Problem (ii) is the content of the next result:

Proposition 6 Let $h$ be a holomorphic mapping on $\mathcal{B}_{R}$ and assume that

$$
N_{R}(\theta):=\sup _{\|x\|<R} \operatorname{Re}\left\langle e^{i \theta} h(x), x^{*}\right\rangle<\infty .
$$

Then

$$
\sup _{\|x\|=r} \operatorname{Re}\left\langle h(x), x^{*}\right\rangle \leq \mathcal{F}_{1}(r, \theta),
$$

where

$$
\begin{aligned}
\mathcal{F}_{1}(r, \theta):= & \sup _{\|x\|=r} \operatorname{Re}\left(\left\langle h(0), x^{*}\right\rangle-\frac{r^{2}}{R^{2}} \overline{\left\langle e^{i \theta} h(0), x^{*}\right\rangle}\right) \\
& +r^{2}\left[L+\mathcal{L}(\theta, r)\left(\frac{1}{R^{2}} N_{R}(\theta)-l(\theta)\right)\right]
\end{aligned}
$$


with

$$
\mathcal{L}(\theta, r)=\frac{2 r(R-r \cos \theta)}{R^{2}-r^{2}}, \quad l(\theta):=\inf _{\|u\|=1} \operatorname{Re}\left\langle e^{i \theta} h^{\prime}(0) u, u^{*}\right\rangle,
$$

and $L:=\sup _{\|u\|=1} \operatorname{Re}\left\langle h^{\prime}(0) u, u^{*}\right\rangle \leq \frac{N_{R}}{R^{2}}$.

Proof. Let $g: \Delta_{R} \rightarrow \mathbb{C}$ be a holomorphic function. By the Kresin-Maz'ya inequalities (see [16]), for all $\zeta \in \Delta_{R}$ with $|\zeta|=r<R$, we have

$$
\operatorname{Re}\left(e^{i \theta}(g(\zeta)-g(0))\right) \leq \mathcal{L}(\theta, r)\left[\sup _{|\xi|<R} \operatorname{Re} g(\xi)-\operatorname{Re} g(0)\right] .
$$

Now, given a holomorphic function $f: \Delta_{R} \rightarrow \mathbb{C}$, let $g$ be the holomorphic function defined in (7). By (8),

$$
\limsup _{r \rightarrow R^{-}} \max _{|\zeta|=r} \operatorname{Re} g(\zeta) \leq \frac{1}{R^{2}} \sup _{|\zeta|<R} \operatorname{Re} f(\zeta) \bar{\zeta} .
$$

Hence, applying (111) to the function $g$, for all $\zeta$ such that $|\zeta|=r \in(0, R)$, we obtain

$$
\begin{aligned}
& \operatorname{Re} e^{i \theta} f(\zeta) \bar{\zeta} \leq \operatorname{Re} e^{i \theta}\left(f(0)-\frac{1}{R^{2}} \overline{f(0)} r^{2}\right) \\
& \quad+r^{2}\left[\operatorname{Re}\left(f^{\prime}(0)\left(e^{i \theta}-\mathcal{L}(\theta, r)\right)\right)+\frac{1}{R^{2}} \mathcal{L}(\theta, r) \sup _{|\xi|<R} \operatorname{Re} f(\xi) \bar{\xi}\right] .
\end{aligned}
$$

Let now $u \in X,\|u\|=1$. Fix $u^{*} \in J(u)$ and consider the holomorphic function $f: \Delta_{R} \rightarrow \mathbb{C}$ defined by $f(\zeta)=\left\langle h(\zeta u), u^{*}\right\rangle$. Applying (12) to such an $f$ and taking into account (8), we can easily finish the proof of this proposition.

Remark 7 The proofs of Propositions 4 and 6 we gave in the original manuscript were more involved and did not rely immediately on the Hadamard-BorelCarathéodory-Kresin-Maz'ya inequalities. We thank one of the anonymous referees for suggesting the shorter proofs contained here.

Remark 8 Let $h$ be a holomorphic mapping on $\mathcal{B}_{R}$ and assume that $N_{R}<+\infty$. Suppose that $h(0)=0$. From the proof of Proposition 6, using (12) with $\theta=0$, it follows that for all $x \in \mathcal{B}_{R}$ and $x^{*} \in J(x)$,

$$
\operatorname{Re}\left\langle h(x), x^{*}\right\rangle \leq \operatorname{Re}\left[\left\langle h^{\prime}(0) x, x^{*}\right\rangle(1-\mathcal{L}(0, r))\right]+\frac{\|x\|^{2}}{R^{2}} \mathcal{L}(0, r) N_{R} .
$$

As we have already mentioned, sometimes it is more convenient to study growth estimates for the function

$$
M_{r}(\theta)=\sup _{\|x\|=r} \operatorname{Re}\left\langle e^{i \theta}(h(x)-h(0)), x^{*}\right\rangle
$$

with respect to $r \in(0, R)$. Of course, this can be done by using Proposition 6 , However, in the same spirit of the previous proof, but using a different auxiliary 
function $f$, one can obtain slightly better estimates. As a matter of notation, if $h: \mathcal{B}_{R} \rightarrow X$ is holomorphic, $r \in(0, R)$ and $\theta \in \mathbb{R}$, let

$$
L(\theta):=\sup _{\|u\|=1} \operatorname{Re}\left\langle e^{i \theta} h^{\prime}(0) u, u^{*}\right\rangle
$$

and

$$
m_{r}(\theta):=\inf _{\|x\|=r} \operatorname{Re}\left\langle e^{i \theta}(h(x)-h(0)), x^{*}\right\rangle .
$$

Moreover, $M_{R}(\theta):=\sup _{\|x\|<R} \operatorname{Re}\left\langle e^{i \theta}(h(x)-h(0)), x^{*}\right\rangle$, and similarly we define $m_{R}(\theta)$. The symbols $\mathcal{L}(\theta, r)$ and $l(\theta)$ were introduced in (10). With these notations at hand we can state and prove the following result:

Proposition 9 Let $h$ be a holomorphic mapping on the ball $\mathcal{B}_{R}$ in $X$. Given $\theta \in \mathbb{R}, R>0$ and $r \in(0, R]$, we have

$$
m_{r}(\theta) \leq r^{2} l(\theta) \leq r^{2} L(\theta) \leq M_{r}(\theta) .
$$

Moreover, for all $x \in \mathcal{B}_{R}$ such that $\|x\|=r<R$ and $x^{*} \in J(x)$ we have

$$
\begin{aligned}
& r^{2}\left(l(0)+\mathcal{L}(\theta, r)\left(\frac{m_{R}(\theta)}{R^{2}}-L(\theta)\right)\right) \leq \operatorname{Re}\left\langle(h(x)-h(0)), x^{*}\right\rangle \\
& \quad \leq r^{2}\left[\mathcal{L}(\theta, r)\left(\frac{M_{R}(\theta)}{R^{2}}-l(\theta)\right)+L(0)\right] .
\end{aligned}
$$

Proof. The proof is a slight modification of the proof of Proposition 6 plus the lower bound estimates given by the Kresin-Maz'ya inequalities.

If $f: \Delta_{R} \rightarrow \mathbb{C}$ is a holomorphic function, we consider the auxiliary holomorphic function $g: \Delta_{R} \rightarrow \mathbb{C}$ defined by

$$
g(\zeta):= \begin{cases}e^{i \theta} \frac{f(\zeta)-f(0)}{\zeta} & \zeta \neq 0, \\ e^{i \theta} f^{\prime}(0) & \zeta=0 .\end{cases}
$$

Applying (11) with $-\theta$ instead of $\theta$ to the function $g$, and taking into account that $\mathcal{L}(\theta, r)=\mathcal{L}(-\theta, r)$, for $\zeta \in \Delta_{R},|\zeta|=r<R$, we obtain

$$
\begin{aligned}
\operatorname{Re}[f(\zeta)-f(0)] \bar{\zeta} \leq & r^{2}\left(\mathcal{L}(\theta, r) \frac{1}{R^{2}} \sup _{|\xi|<R} \operatorname{Re}\left(e^{i \theta}[f(\zeta)-f(0)] \bar{\zeta}\right)\right. \\
& \left.+\operatorname{Re}\left[\left(e^{-i \theta}-\mathcal{L}(\theta, r)\right) e^{i \theta} f^{\prime}(0)\right]\right)
\end{aligned}
$$

Let now $u \in X,\|u\|=1$. Fix $u^{*} \in J(u)$ and consider the holomorphic function $f: \Delta_{R} \rightarrow \mathbb{C}$ defined by $f(\zeta)=\left\langle h(\zeta u), u^{*}\right\rangle$. Applying (16) to $f$, we obtain

$$
M_{r}(0) \leq r^{2}\left[\mathcal{L}(\theta, r)\left(\frac{M_{R}(\theta)}{R^{2}}-l(\theta)\right)+L(0)\right],
$$

which gives the upper bound in (14). 
Note that for each $\theta \in \mathbb{R}$ and $x \in \mathcal{B}_{R}$ such that $\|x\|=r \leq R, x=\zeta u,|\zeta|=r$, $x^{*} \in J(x)$, it follows from the maximum principle for harmonic functions that

$$
\begin{aligned}
\operatorname{Re}\left\langle e^{i \theta} h^{\prime}(0) u, u^{*}\right\rangle & =\operatorname{Re} e^{i \theta} f^{\prime}(0)=\operatorname{Re} g(0) \leq \max _{|\zeta|=r} \operatorname{Re} g(\zeta) \\
& \leq \frac{1}{r^{2}} \sup _{\|x\|=r} \operatorname{Re}\left\langle e^{i \theta}[h(x)-h(0)], x^{*}\right\rangle=\frac{1}{r^{2}} M_{r}(\theta),
\end{aligned}
$$

which implies that, for each $r \in(0, R)$,

$$
l(\theta) \leq L(\theta) \leq \frac{M_{r}(\theta)}{r^{2}},
$$

giving the upper estimates in (13).

In order to get the lower estimates, let us recall the Kresin-Maz'ya lower bound (see [16]). Let $g: \Delta_{R} \rightarrow \mathbb{C}$ be a holomorphic function. Then, given $\theta \in \mathbb{R}$, for all $\zeta \in \Delta_{R}$ with $|\zeta|=r<R$ we have

$$
\operatorname{Re}\left(e^{i \theta}(g(\zeta)-g(0))\right) \geq \mathcal{L}(\theta, r)\left[\inf _{|\xi|<R} \operatorname{Re} g(\xi)-\operatorname{Re} g(0)\right] .
$$

Then, one can argue exactly as before, just replacing (11) with (18).

Corollary 10 (Rigidity property) Let $h: \mathcal{B}_{R} \rightarrow X$ be holomorphic and assume that for some $\theta \in \mathbb{R}$, one of the following equalities holds:

$$
M_{R}(\theta)=R^{2} l(\theta)
$$

or

$$
m_{R}(\theta)=R^{2} L(\theta) .
$$

Then the second equality holds too and $h$ is, in fact, an affine mapping: $h(x)=$ $h^{\prime}(0) x+h(0)$. In particular, if $h(0)=0$, then $h$ is a linear operator, that is, $h(x)=h^{\prime}(0) x$.

Proof. If the first equality holds, then $l(\theta)=L(\theta)$ by (13), and hence by (14), $l(0)=L(0)$, whence by (14),

$$
\operatorname{Re}\left\langle(h(x)-h(0)), x^{*}\right\rangle \leq r^{2} l(0)=\inf _{\|u\|=1} \operatorname{Re}\left\langle h^{\prime}(0) u, u^{*}\right\rangle,
$$

where $\|x\|=r<R$.

Since $\left\langle h^{\prime}(0) u, u^{*}\right\rangle=g(0)$, where, as above, $g(\zeta)=\frac{1}{\zeta}\left\langle h(\zeta u)-h(0), u^{*}\right\rangle$, this implies that

$$
\operatorname{Re} g(\zeta) \leq \operatorname{Re} g(0), \quad|\zeta|<R .
$$

Hence, by the maximum principle for harmonic functions, $\operatorname{Re} g(\zeta)=\operatorname{Re} g(0)$ in $\Delta_{R}$, which means that

$$
\left\langle h(x)-h(0)-h^{\prime}(0) x, x^{*}\right\rangle=0
$$

for all $x \in \mathcal{B}_{R}$ and $x^{*} \in J(x)$.

Now it follows from Proposition 1 in [12] that $h(x)=h(0)+h^{\prime}(0) x$ and we are done.

Similarly, one can get the same conclusion if the second equality holds. 
Remark 11 Let $h: \mathcal{B}_{R} \rightarrow X$ be holomorphic. Let $M_{R}=M_{R}(0)$ and $u \in X$, $\|u\|=1$. Fix $u^{*} \in J(u)$ and consider the holomorphic function $f: \Delta_{R} \rightarrow \mathbb{C}$ defined by $f(\zeta)=\left\langle h(\zeta u), u^{*}\right\rangle$. Equation (6) applied to the function $g$ defined in (7) implies that

$$
r^{2} L \leq M_{r} \leq \frac{R-r}{R+r} L+\frac{2 r}{R+r} M_{R} .
$$

Also, by the same token and using the classical Littlewood two-sided estimates (see [16]), one can establish another lower bound for $M_{r}$. Namely,

$$
r^{2} p(r) \leq M_{r},
$$

where

$$
p(r)=\frac{R+r}{R-r} \cdot L-\frac{2 r}{R-r} \cdot \frac{1}{R^{2}} M_{R} .
$$

However, the left-hand side inequality in (19) is better. Indeed, this inequality says that for all $r \in(0, R], L \leq \frac{M_{r}}{r^{2}}$, and, in particular,

$$
L \leq \frac{M_{R}}{R^{2}},
$$

which, in turn, implies that for $r \in(0, R)$,

$$
p(r) \leq \frac{R+r}{R-r} \cdot L-\frac{2 r}{R-r} \cdot L=L .
$$

An immediate consequence of Proposition 4 is the following growth estimate for the numerical radius which, in its turn implies, by Proposition 1 in [12, an estimate for the growth of the norm of $h$.

Corollary 12 Let $h: \mathcal{B}_{R} \rightarrow X$ be a holomorphic mapping on $\mathcal{B}_{R}$ with $h(0)=0$ and assume that $N_{R}(h):=\sup _{\|x\|<R} \operatorname{Re}\left\langle h(x), x^{*}\right\rangle$ is finite. Then for each $r \in$ $(0, R)$, the values $\left|V_{r}(h)\right|=\sup _{\|x\|<r}\left|\left\langle h(x), x^{*}\right\rangle\right|$ and $W_{r}(h)=\sup _{\|x\| \leq r}\|h(x)\|$ are finite. Moreover,

$$
\left|V_{r}(h)\right| \leq \frac{r^{2}}{R+r}\left[(R-r) L+\frac{2 r N_{R}}{R^{2}}\right],
$$

and

$$
\left|W_{r}(h)\right| \leq \frac{2 R^{2}}{(R-r)^{2}}\left|V_{R}(h)\right| .
$$

In particular, if the closed convex hull of the numerical range of a holomorphic mapping on the unit ball is not the whole complex plane, then it has unit radius of boundedness. 


\section{Nonlinear resolvents of holomorphic mappings and semi-complete vector fields}

We start this section with the following notions.

Definition 13 Let $\mathcal{D}$ be a domain in $X, \mathcal{D} \ni 0$, and let $h: \mathcal{D} \rightarrow X$ be holomorphic. We define the resolvent set $\rho(h) \subseteq \mathbb{C}$ of $h$ to be the set of those complex numbers $\lambda \in \mathbb{C}$ for which there is an open set $\mathcal{D}_{\lambda} \subseteq \mathcal{D}, \mathcal{D}_{\lambda} \ni 0$, such that $\lambda I-h$ is holomorphically invertible on $\mathcal{D}_{\lambda}$. The complement $\sigma(h)$ of $\rho(h)$ is called the spectrum of $h$.

In other words, the spectrum $\sigma(h)$ of $h$ consists of those $\lambda \in \mathbb{C}$ such that it is not possible to find an open subset $\mathcal{D}_{\lambda}$ and a neighborhood $V_{\lambda} \subseteq(\lambda I-h) \mathcal{D}_{\lambda}$, $V_{\lambda} \ni-h(0)$, such that $(\lambda I-h)^{-1}$ is a well-defined holomorphic mapping on $V_{\lambda}$ with values in $\mathcal{D}_{\lambda}$.

Remark 14 It was shown by L. A. Harris that $\sigma(h)=\sigma\left(h^{\prime}(0)\right)$ and, respectively, $\rho(h)=\rho\left(h^{\prime}(0)\right)($ see [12] $)$.

We set $\Re(\lambda, h):=(\lambda I-h)^{-1}$ whenever it exists on an open domain $V_{\lambda}(\ni$ $-h(0))$, and $V_{\lambda}$ is called the domain of the resolvent $\Re(\lambda, h)$.

We will see below that the properties of the resolvent set of a holomorphic mapping as well as the domain of definition of its resolvent can be described in terms of the numerical range of the given mapping.

As we have already mentioned in Section 1, the problem of finding a domain $V_{\lambda}$ for the existence of the resolvent $\Re(\lambda, h)$ is related to the problem of local and global descriptions of semi-complete vector fields. This observation is based on the following fact (see, for example, [20]).

Criterion 15 Let $\mathcal{D}$ be a bounded and convex domain in a complex Banach space $X$, and let $h: \mathcal{D} \rightarrow X$ be a holomorphic mapping on $\mathcal{D}$. Then for some real number $\mu$, the mapping $\mu I-h$ is a semi-complete vector field on $\mathcal{D}$ if and only if the equation

$$
(\lambda I-h)(x)=(\lambda-\mu) y
$$

has a unique solution $x=\Re(\lambda, h) \circ((\lambda-\mu) I)(y)$ for each $y \in \mathcal{D}$.

We call the mapping $\Phi_{\lambda}:=\Re(\lambda, h) \circ((\lambda-\mu) I): \mathcal{D} \rightarrow \mathcal{D}$ the associated resolvent mapping of $h-\mu I$.

As above, let $h$ be a holomorphic mapping on the ball $\mathcal{B}_{R}$ and assume that

$$
N_{R}=\sup _{\|x\|<R} \operatorname{Re}\left\langle h(x), x^{*}\right\rangle<\infty
$$

Consider the resolvent equation

$$
\lambda x-h(x)=z, \quad z \in X
$$


For a fixed $r \in(0, R)$, we would like to find conditions which ensure that (20) has a unique solution $x=x(z) \in \mathcal{B}_{r}$. To this end, we define the mapping $G: \mathcal{B}_{r} \rightarrow X$ by the formula

$$
G(x):=z-\lambda x+h(x) .
$$

Then for every $x \in \partial \mathcal{B}_{r}$ and $x^{*} \in J(x), \operatorname{Re}\left\langle G(x), x^{*}\right\rangle \leq\|z\| r-r^{2} \operatorname{Re} \lambda+r \omega(r)$, where, by Proposition 4 .

$$
\omega(r)=\frac{1}{r} \mathcal{F}(r)=\|h(0)\|\left(1-\frac{r^{2}}{R^{2}}\right)+\frac{r}{R+r}\left[(R-r) L+2 r \cdot \frac{N_{R}}{R^{2}}\right]
$$

with $L=\sup _{\|u\|=1} \operatorname{Re}\left\langle h^{\prime}(0) u, u^{*}\right\rangle$.

Hence, if

$$
\|z\|+\omega(r)<r \operatorname{Re} \lambda
$$

then we obtain the inequality

$$
\sup _{\|x\|=r} \operatorname{Re}\left\langle G(x), x^{*}\right\rangle<0,
$$

which implies the existence and the uniqueness of solutions to (20) (see [14]).

Let now $\mu=\mu(r), r \in[0, R]$, be a finite real-valued function and assume that $\lambda \in \mathbb{R}, \lambda>\mu(r)$.

Solving (22) with $z=(\lambda-\mu) y$, where $y \in \mathcal{B}_{r}$, we get the condition

$$
\lambda>\max \{\eta(r), \mu(r)\},
$$

where

$$
\eta(r)=\frac{\omega(r)-\mu(r)\|y\|}{r-\|y\|} .
$$

Note that $\mu(r) \geq \eta(r)$ if and only if $\mu(r) \cdot r \geq \omega(r)$. In particular, if $\mu(r) \cdot r=$ $\omega(r)$, then

$$
\mu(r)=\eta(r) .
$$

Finally, one can use classical calculus to investigate the function $\mu(r)=\frac{1}{r} \omega(r)$. Denote $b:=\frac{N_{R}}{R^{2}}, c:=\|h(0)\|$, and consider the real-valued function

$$
\mu(r)=\frac{R-r}{R+r} L+\frac{2 r}{R+r} b+\frac{c}{r}\left(1-\frac{r^{2}}{R^{2}}\right), \quad r \in(0, R) .
$$

If $c=0$, then $\mu(r)$ has no strict minimum in $(0, R)$. Indeed, in the case $c=0$ and $b=L, \mu$ is the constant function $\mu(r)=L$, and if $c=0$ and $b \neq L$, the derivative $\mu^{\prime}(r)=\frac{2 R(b-L)}{(R+r)^{2}}>0$, so $\mu(r)$ is strictly increasing with $\mu(0)=L$ and $\mu(R)=b$.

Otherwise, if $c \neq 0$, then the derivative

$$
\mu^{\prime}(r)=\frac{2 R(b-L)}{(R+r)^{2}}-c \frac{r^{2}+R^{2}}{R^{2} r^{2}}=-\frac{c\left(R^{2}+r^{2}\right)^{2}+2 c r R\left(R^{2}+r^{2}\right)-2 R(b-L)(R r)^{2}}{(R+r)^{2} R^{2} r^{2}}
$$


equals zero if and only if $R^{2}+r^{2}=\beta R r$, where $\beta=\frac{\sqrt{c^{2}+2 R(b-L) c}-c}{c}$.

If $\beta \leq 2$, then the equation $R^{2}+r^{2}=\beta R r$ has no solution in $(0, R)$. If $\beta>2$ or, which is one and the same, $R(b-L)>4 c$, then it has the unique solution $r_{*}=R \frac{\beta-\sqrt{\beta^{2}-4}}{2}$ in $(0, R)$ and since $\mu^{\prime}(R)>0, \mu$ has a minimum at $r_{*}$.

Thus we have arrived at the following result.

Proposition 16 Let $h: \mathcal{B}_{R} \rightarrow X$ be holomorphic in $\mathcal{B}_{R}$ and let

$$
N_{R}=\sup _{\|x\|=R} \operatorname{Re}\left\langle h(x), x^{*}\right\rangle<\infty .
$$

The following assertions hold:

(i) If $\omega(r)$ is given by (21), then for each $z \in X$ and all $\lambda \in \mathbb{C}$ such that

$$
\operatorname{Re} \lambda>\frac{1}{r}[\omega(r)+\|z\|],
$$

the equation

$$
(\lambda I-h)(x)=z
$$

has a unique solution $x=\Re(\lambda, h)(z) \in \mathcal{B}_{r}$.

(ii) In particular, if we define $\mu(=\mu(r))=\frac{1}{r} \omega(r)$, then for each $\lambda>\mu$, the equation

$$
(\lambda I-h)(x)=(\lambda-\mu) y
$$

has a unique solution $x=x(y) \in \mathcal{B}_{r}$ whenever $y \in \mathcal{B}_{r}$.

(iii) If $h(0)=0$, then the function $\mu=\mu(r)$ has no strict minimum in the interval $(0, R)$. In particular, it is either the constant function $\mu(r)=L$ if $N_{R}=R^{2} L$ or a strictly increasing function otherwise.

(iv) If $h(0) \neq 0$, then the function $\mu=\mu(r)$ has a minimum at the point $r_{*}=R \frac{\beta-\sqrt{\beta^{2}-4}}{2}$ in $(0, R)$, where $\beta=\frac{\sqrt{c^{2}+2 R(b-L) c}-c}{c}$, if and only if

$$
R\left(\frac{N_{R}}{R^{2}}-L\right)>4\|h(0)\| .
$$

We recall that a mapping $-h: \mathcal{B}_{R} \rightarrow X$ is said to be a locally semi-complete vector field if there is $r \in(0, R)$ such that $-h$ is semi-complete on $\mathcal{B}_{r}$. It follows from the above criterion that $-h$ is a locally semi-complete vector field whenever the function $\mu(r)$ vanishes or is negative in the interval $(0, R)$.

The simplest situation occurs when $h(0)=0$. Note that a necessary condition for $\mu(r)$ to vanish at some point of the interval $(0, R)$ is that $L<0$.

Thus we get the following conclusion.

Corollary 17 Let $h: \mathcal{B}_{R} \rightarrow X$ be holomorphic in $\mathcal{B}_{R}$ with $h(0)=0$, and let

$$
N_{R}=\sup _{\|x\|<R} \operatorname{Re}\left\langle h(x), x^{*}\right\rangle<\infty .
$$


Then $-h$ is a locally semi-complete vector field if and only if the following condition holds:

$$
L=\sup _{\|u\|=1} \operatorname{Re}\left\langle h^{\prime}(0) u, u^{*}\right\rangle<\min \left\{0, \frac{N_{R}}{R^{2}}\right\} .
$$

In this case $-h$ is semi-complete on each $\mathcal{B}_{r}$ with $r \in\left(0, \frac{-R^{3} L}{2 N_{R}-L R^{2}}\right)$.

Since in general when $h(0) \neq 0$ the equation $\mu(r)=0$ is equivalent to a third order algebraic equation it can be seen by using Vieta's formulas that under condition (26) this equation has three positive roots $\left\{r_{i}\right\}_{i=1}^{3}$ such that $0<r_{1}<r_{*}<r_{2} \leq R<r_{3}$ if and only if $\mu\left(r_{*}\right)<0$.

Theorem 18 Let $h: \mathcal{B}_{R} \rightarrow X$ be holomorphic in $\mathcal{B}_{R}$ with $h(0) \neq 0$, and let

$$
N_{R}=\sup _{\|x\|<R} \operatorname{Re}\left\langle h(x), x^{*}\right\rangle<\infty .
$$

If $R\left(\frac{N_{R}}{R^{2}}-L\right)>4\|h(0)\|$ and $\mu\left(r_{*}\right)<0$, where $r_{*}=R \frac{\beta-\sqrt{\beta^{2}-4}}{2} \in(0, R)$, with $\beta=\frac{\sqrt{c^{2}+2 R(b-L) c}-c}{c}$, then $-h$ is semi-complete on each $\mathcal{B}_{r}$ with $r \in\left(r_{1}, r_{2}\right)$, where $r_{1} \stackrel{c}{<} r_{2}$ are the roots of the equation $\mu(r)=0$ in $(0, R]$.

Remark 19 One can find the values of $r_{1}$ and $r_{2}$ by the formulas

$$
r_{1}=-2 \sqrt{Q} \cos \phi-\frac{m}{3}, \quad r_{2}=-2 \sqrt{Q} \cos \left(\phi-\frac{2 \pi}{3}\right)-\frac{m}{3},
$$

where $m=\frac{R(R L-2 R b+c)}{c}, \phi=\frac{1}{3} \arccos \frac{A}{\sqrt{Q^{3}}}, Q=\frac{c m^{2}+3 L R^{3}+3 c R^{2}}{9 c}$ and $A=\frac{2 c m^{3}+9 m\left(L R^{3}+c R^{2}\right)-27 c R^{3}}{54 c}$.

The situation becomes more transparent if we assume that $N_{R}=0$. In this case one of the roots is equal to $R$ and, actually, condition (26) already yields $\mu\left(r_{*}\right)<0$; hence $0<r_{1}<r_{2}=R$. Since this situation is of some special interest in the open unit ball $\mathcal{B}$ and has applications to fixed point theory, we will describe it separately.

Corollary 20 Let $-h$ be a semi-complete vector field on the open unit ball $\mathcal{B}$ in $X$. Then $L=\sup _{\|u\|=1} \operatorname{Re}\left\langle h^{\prime}(0) u, u^{*}\right\rangle \leq 0$, that is, the linear mapping $-h^{\prime}(0)$ is also semi-complete on each ball $\mathcal{B}_{R}, R>0$. Moreover, if $L$ satisfies the stronger condition

$$
L+4\|h(0)\|<0,
$$

then the following assertions hold. 
(i) Let

$$
r_{1}=\frac{1}{2\|h(0)\|}[-(2\|h(0)\|+L)-\sqrt{(L+4\|h(0)\|) L}](<1)
$$

be the smaller root of the quadratic equation

$$
\|h(0)\|(1+r)^{2}+L r=0 .
$$

Then for each $r \in\left[r_{1}, 1\right]$, the mapping $-h$ is semi-complete on the ball $\mathcal{B}_{r}$.

(ii) The mapping $h$ has a unique null point $x_{0}$ in $\mathcal{B}$ with $\left\|x_{0}\right\| \leq r_{1}$.

(iii) If the family $\left\{\Phi_{\lambda}\right\}_{\lambda \geq 0}$ is defined by using the resolvent $\Re(\lambda, h)$,

$$
\Phi_{\lambda}:=\Re(\lambda, h) \circ(\lambda I):=(\lambda I-h)^{-1} \circ(\lambda I),
$$

then for each $\lambda>0$, the iterates $\left\{\Phi_{\lambda}^{n}\right\}_{n=1}^{\infty}$ converge to the constant mapping taking the value $x_{0}$, uniformly on each ball strictly inside $\mathcal{B}$.

Proof. First we note that the inequality $\mu(r) \leq 0$ is equivalent to the inequality $\omega(r)=r \mu(r)=\frac{1-r}{1+r}\left(\|h(0)\|(1+r)^{2}+L r\right) \leq 0$. Therefore, $\mu(r)$ is negative on the interval $\left[r_{1}, 1\right)$ with $r_{1}<1$ if and only if condition (27) holds. In this case $r_{1}$ is the smaller root of equation (28). This prove assertion (i). To prove assertions (ii) and (iii) we return to equation (20) and recall that it has a unique solution if and only if condition (23) holds. Since for each $r \in\left(r_{1}, 1\right), \omega(r)$ is negative, one can set $\lambda=0$ and $z=0$ to obtain the existence and uniqueness of the solution of the equation $h(x)=0$. On the other hand, setting in (20) $z=\lambda y$, $\|y\|=r(1-\mu(r)):=R$, we see that for each $r \in\left(r_{1}, 1\right)$, inequality (23) holds for each $\lambda>0$. This means that for such $\lambda$ the mapping $\Phi_{\lambda}=(\lambda I-h)^{-1} \circ(\lambda I)$ is well defined on the ball of radius $R$ and maps it into the smaller ball of radius $r \in\left(r_{1}, 1\right)$. It then follows from the Earle-Hamilton Theorem [6] that $\Phi_{\lambda}$ has a unique fixed point $x(\lambda) \in \mathcal{B}_{r}$ and that its iterates $\left\{\Phi_{\lambda}^{n}\right\}_{n=1}^{\infty}$ converge to $x(\lambda)$, uniformly on each ball strictly inside $\mathcal{B}_{R}$. Since $\mu(r) \rightarrow 0$ as $r \rightarrow 1^{-}$, we see that $R \rightarrow 1$. Hence this convergence is uniform on each ball strictly inside $\mathcal{B}$. Finally, we note that since $h\left(x_{0}\right)=0, \Phi_{\lambda}\left(x_{0}\right)=x_{0}$. Hence $x(\lambda)=x_{0}$ does not depend on $\lambda>0$ because of the uniqueness property. The proof is complete.

Corollary 21 Let $F: \mathcal{B} \rightarrow \mathcal{B}$ be a holomorphic self-mapping of $\mathcal{B}$ and assume that

$$
L_{F}:=\sup _{\|u\|=1} \operatorname{Re}\left\langle F^{\prime}(0) u, u^{*}\right\rangle<1-4\|F(0)\| .
$$

Then $F$ has a unique fixed point $x_{0}$ in $\mathcal{B}$ with $\left\|x_{0}\right\| \leq r_{1}$, where $r_{1}$ is the fixed point in the interval $(0,1)$ of the scalar mapping $\phi(r)=\|F(0)\|(1+r)^{2}+r L_{F}$.

Remark 22 Inequality (29) reminds us of the well-known one-dimensional Schwarz inequality

$$
\left|F^{\prime}(0)\right| \leq 1-|F(0)|^{2} .
$$

However, even in the one-dimensional case the last inequality does not imply the existence of an interior fixed point of $F$. 
We conclude this section with a result on the holomorphic extension of the associated resolvent mapping to key domains of the complex plane.

Theorem 23 Let $h$ be a semi-complete vector field on the open unit ball $\mathcal{B}$ in $X$ with $h(0)=0$ and $h^{\prime}(0)=-I$. Then for each $r \in(0,1)$ and $\lambda \in \Omega$, where $\Omega=\Omega_{1} \cup \Omega_{2}$ is a key domain defined by the disc $\Omega_{1}=\left\{\lambda \in \mathbb{C}:|\lambda|<\frac{1}{2} \frac{1-r}{1+r}\right\}$ and the sector $\Omega_{2}=\left\{\lambda \in \mathbb{C}, \lambda \neq 0:|\arg \lambda|<\arcsin \frac{1-r^{2}}{1+r^{2}}\right\}$, the associated resolvent mapping

$$
\Phi_{\lambda}=(\lambda I-h)^{-1} \circ(\lambda I)
$$

is a self-mapping of the ball $\mathcal{B}_{r}$. Moreover, this mapping is holomorphic on $\Omega \times \mathcal{B}_{r}$.

Proof. First we show that $\Phi_{\lambda}$ is well defined on $\Omega_{1}$ and maps $\mathcal{B}_{r}$ into itself, or which is one and the same, that the equation

$$
(\lambda I-h)(x)=\lambda y
$$

has a unique solution $x \in \mathcal{B}_{r}$ for each $\lambda \in \Omega_{1}$ and $y \in \mathcal{B}_{r}$. To this end, we consider the mapping

$$
G(x)=\lambda y-\lambda x+h(x)
$$

and show that

$$
\sup _{\|x\|=r} \operatorname{Re}\left\langle G(x), x^{*}\right\rangle<0 .
$$

Indeed, it follows from Proposition 4 that under our assumptions,

$$
\begin{aligned}
\sup _{\|x\|=r} \operatorname{Re}\left\langle G(x), x^{*}\right\rangle & \leq \sup _{\|x\|=r} \operatorname{Re}\left\langle\lambda(y-x), x^{*}\right\rangle+\frac{r^{2}(1-r)}{1+r} \sup _{\|u\|=1} \operatorname{Re}\left\langle h^{\prime}(0) u, u^{*}\right\rangle \\
& \leq \sup _{\|u\|=1}|\lambda|\|y-x\| r-\frac{r^{2}(1-r)}{1+r}<\frac{1}{2} \frac{1-r}{1+r} 2 r^{2}-\frac{r^{2}(1-r)}{1+r}=0 .
\end{aligned}
$$

Thus $G$ has a unique null point $x=\Phi_{\lambda}(y)$ in $\mathcal{B}_{r}$, as required.

Now assume that for some $r \in(0,1)$, the complex number $\lambda=|\lambda| e^{i \theta} \in \Omega_{2}$ is given. Then equation (30) can be rewritten as

$$
\left(|\lambda| I-e^{-i \theta} h\right)(x)=|\lambda| y .
$$

In its turn, the last equation has a unique solution $x \in \mathcal{B}_{r}$ for each $\lambda \in \Omega_{2}$ and $y \in \mathcal{B}_{r}$ whenever the mapping $e^{-i \theta} h$ is semi-complete on $\mathcal{B}_{r}$ or, which is one and the same,

$$
\sup _{\|x\|=r} \operatorname{Re}\left\langle e^{-i \theta} h(x), x^{*}\right\rangle \leq 0 .
$$

It follows from Proposition 6 that

$$
\sup _{\|x\|=r} \operatorname{Re}\left\langle e^{-i \theta} h(x), x^{*}\right\rangle \leq r^{2}\left(\frac{2 r(1-r \cos \theta)}{1-r^{2}}-\cos \theta\right)=\frac{r^{2}}{1-r^{2}} \varphi(r) \leq 0,
$$


as long as $|\theta|=|\arg \lambda|<\arcsin \frac{1-r^{2}}{1+r^{2}}$, and we are done. To finish the proof we just note that since the point $x=0$ is a regular null point of the mapping $G$ defined by (31) $\left(G^{\prime}(0)=-I\right)$, it follows from a version of the global implicit function theorem in [15] (see also Lemma25] below) that the solution $x=\Phi_{\lambda}(y)$ of the equation $G(x)(=G(x, \lambda, y))=0$ holomorphically depends on $(\lambda, y) \in$ $\Omega \times \mathcal{B}_{r}$. This completes our proof.

Remark 24 Actually, as we will see below (see Section 4), the solution $r=$ $r(\theta)=\frac{1-|\sin \theta|}{\cos \theta}$ of the equation $\varphi(r)=0$, where $\varphi(r)=2 r(1-r \cos \theta)-$ $\cos \theta\left(1-r^{2}\right)$ is defined in (32), determines the radius of spirallikeness for starlike mappings defined on the unit ball $\mathcal{B}$.

\section{Bloch radii}

Let $F: \mathcal{B} \rightarrow X$ be such that $F(0)=0$ and $F^{\prime}(0)$ is an invertible operator on $X$. In other words, $F$ is locally biholomorphic around the origin.

One says that the positive numbers $r$ and $\rho$ are Bloch radii for $F$ if $F\left(\mathcal{B}_{r}\right) \supseteq$ $\mathcal{B}_{\rho}$ and $F^{-1}: \mathcal{B}_{\rho} \rightarrow \mathcal{B}_{r}$ is a well-defined holomorphic mapping on $\mathcal{B}_{\rho}$.

A deficiency of this definition is that the pair $(r, \rho)$ is not uniquely defined. If, for example, we find the maximal $\rho$ for which $F^{-1}$ is holomorphic on $\mathcal{B}_{\rho}$, then for each $\widetilde{r} \in[r, 1]$, the pair $(\widetilde{r}, \rho)$ constitutes Bloch radii. However, in this case it is often desirable to find the minimal $r$ for which $F\left(\mathcal{B}_{r}\right) \supseteq \mathcal{B}_{\rho}$.

Sometimes it is preferable to find a number $0<r_{*} \leq 1$ and a continuous function $\rho(r)$ on $\left[0, r_{*}\right]$ (if it exists) such that all the pairs $(r, \rho(r))$ are Bloch radii. In this case, one can investigate the distortion (dilation) coefficient

$$
\varepsilon(r)=\frac{r}{\rho(r)}>0
$$

on the interval $\left[0, r_{*}\right]$ and look for its bounds.

For example, if $F(0)=0$ and $F^{\prime}(0)=I$, then in the one-dimensional case it follows from Koebe's 1/4-theorem that if $r_{*}$ is a radius of univalence of $F$ in $\mathcal{B}$, then $\varepsilon(r)=4$ for each $r \in\left(0, r_{*}\right]$.

In general, under the above normalization, the inverse function theorem shows that Bloch radii exist for $F$. In this case, one can write $F(x)=x-h(x)$, where $h^{\prime}(0)=0$. However, the latter condition is not necessary: one can just require that $I-h^{\prime}(0)$ be an invertible linear operator. In particular, in order to get estimates in terms of the numerical range we can assume that

$$
L=\sup _{\|x\|=1} \operatorname{Re}\left\langle h^{\prime}(0) x, x^{*}\right\rangle<1 .
$$

Consider the equation

$$
x-h(x)=z, \quad z \in X, \quad\|x\|<1 .
$$


Our goal is to find numbers $0<r<1$ and $\rho(=\rho(r))$ such that for all $z \in \mathcal{B}_{\rho}$, equation (33) has a unique solution $x=x(z) \in \mathcal{B}_{r}$, which is holomorphic in $z \in \mathcal{B}_{\rho}$.

If $N=\limsup _{s \rightarrow 1^{-1}} \sup _{\|x\|=1} \operatorname{Re}\left\langle h(s x), x^{*}\right\rangle<\infty$, then one can use Proposition 16 with $R=1$ and $\lambda=1$ to obtain estimates for the Bloch radii. However, one can devise an algorithm for finding lower bounds of Bloch radii under weaker restrictions.

Let us assume that $h(0)=0$ and that for some $\theta \in \mathbb{R}$, the mapping $h$ satisfies the condition

$$
\sup _{x \in B} \operatorname{Re}\left\langle e^{i \theta} h(x), x^{*}\right\rangle=N(\theta)<\infty .
$$

Suppose that the following numbers are given:

$$
L(\theta):=\sup _{\|x\|=1} \operatorname{Re}\left\langle e^{i \theta} h^{\prime}(0) x, x^{*}\right\rangle \quad \text { and } \quad l(\theta):=\inf _{\|x\|=1} \operatorname{Re}\left\langle e^{i \theta} h^{\prime}(0) x, x^{*}\right\rangle .
$$

We let $L:=L(0)$.

Since the Fréchet derivative of a holomorphic mapping is a bounded linear operator, $L(\theta)$ and $l(\theta)$ are finite for all $\theta \in \mathbb{R}$.

We use the following version of the implicit function theorem

Lemma 25 ([18] and [15]) Let $G(=G(x, z))$ be a holomorphic mapping in the domain $\mathcal{D}=\mathcal{B}_{r} \times \mathcal{B}_{\rho}$ with values in $X$ and assume that for each $z \in \mathcal{B}_{\rho}$ and $x=s u,\|u\|=r, 0<s<1$,

$$
\limsup _{s \rightarrow 1^{-}} \operatorname{Re}\left\langle G(s u, z), u^{*}\right\rangle<0 .
$$

Then

(i) for each $z \in \mathcal{B}_{\rho}$, there is a unique solution $x(=x(z)) \in \mathcal{B}_{r}$ of the equation

$$
G(x, z)=0,
$$

which holomorphically depends on $z \in \mathcal{B}_{\rho}$;

(ii) for each $z \in \mathcal{B}_{\rho}$, the linear operator $G_{x}^{\prime}(x(z), z)$ is invertible in $X$.

We now consider the mapping $G: B \times X \rightarrow X$ defined by

$$
G(x, z):=z-x+h(x)
$$

and note that equation (33) is equivalent to

$$
G(x, z)=0 .
$$

In view of Lemma 25, our aim becomes to find $r \in(0,1)$ and $\rho=\rho(r)>0$ such that the following inequality holds whenever $\|x\|=r$ and $\|z\|<\rho(=\rho(r))$ :

$$
\sup _{\|x\|=r} \operatorname{Re}\left\langle G(x, z), x^{*}\right\rangle<0 .
$$


Equation (38) is a sufficient condition for (37) to have a unique solution $x=x(z)$ in the ball $\mathcal{B}_{r}, r \in(0,1)$.

Since

$$
\sup _{\|x\|=r} \operatorname{Re}\left\langle G(x, z), x^{*}\right\rangle \leq\|z\| \cdot r-r^{2}+\sup _{\|x\|=r} \operatorname{Re}\left\langle h(x), x^{*}\right\rangle,
$$

we have, as a matter of fact, to use an appropriate growth estimate for the last term in (39).

Now it follows from Proposition 6 (with $R=1$ ) that

$$
\sup _{\|x\|=r} \operatorname{Re}\left\langle h(x), x^{*}\right\rangle \leq r^{2}[L+\mathcal{L}(\theta, r)(N(\theta)-l(\theta))] .
$$

Returning to (39), we finally get for $\|z\| \leq \rho$ that

$$
\sup _{\|x\|=r} \operatorname{Re}\left\langle G(x, z), x^{*}\right\rangle \leq r[\rho-r(1-L-\delta(\theta) \mathcal{L}(\theta, r))],
$$

where

$$
\delta(\theta):=N(\theta)-l(\theta) \geq 0
$$

by Proposition 9 Moreover, $\delta(\theta)=0$ if and only if $h(x)=h^{\prime}(0) x, x \in \mathcal{B}$, is a restriction of the bounded linear operator $h^{\prime}(0)$ in $X$ (see Corollary 101). So, in this case we have by (41),

$$
\sup _{\|x\|=r} \operatorname{Re}\left\langle G(x, z), x^{*}\right\rangle \leq 0
$$

if

$$
\rho \leq r(1-L), \quad r \in(0,1] .
$$

Thus for each $r \in(0,1]$ and $\rho(r)=r(1-L)$, the pair $(r, \rho(r))$ constitutes Bloch radii, and $\varepsilon(r)=\frac{r}{\rho(r)}=\frac{1}{1-L}$ is a constant function. Obviously, the function $\rho(r)$ is positive on $(0,1]$ and attains its maximum $\rho_{0}=1-L$ at the point $r_{0}=1$ whenever $L<1$.

Now we assume that $\delta(\theta)>0$, that is, $N(\theta)>l(\theta)$. In this case

$$
\sup _{\|x\|=r} \operatorname{Re}\left\langle G(x, z), x^{*}\right\rangle \leq 0
$$

if

$$
\rho<\rho(r):=r(1-L-\delta(\theta) \mathcal{L}(\theta, r)), \quad\|z\|<\rho, \quad\|x\|=r .
$$

It would, of course, be pertinent to look for conditions which ensure that $\rho(r)>0$ for some $r \in(0,1]$ and to find the maximum of this function on this interval.

Writing down explicitly (44), we get

$$
\rho(r)=\frac{r}{1-r^{2}}\left((1-L)\left(1-r^{2}\right)-\delta(\theta) 2 r(1-r \cos \theta)\right) .
$$


As above we assume in the sequel that $L<1$. Since $\rho(0)=0$, we have $\rho^{\prime}(0)=$ $\lim _{r \rightarrow 0^{+}} \frac{\rho(r)}{r}=1-L>0$. In addition, $\lim _{r \rightarrow 1^{-}} \rho(r)=-\infty$ whenever $\theta \neq 0$, and for all $r \in(0,1)$,

$$
\rho^{\prime \prime}(r)=\frac{4 \delta\left(r^{3} \cos \theta-3 r^{2}+3 r \cos \theta-1\right)}{\left(1-r^{2}\right)^{3}}<\frac{-4 \delta}{(1+r)^{3}}<0 .
$$

Thus, again, the condition $L<1$ ensures that $\rho$ has a positive maximum $\rho\left(r_{0}\right)$ at some point $r_{0} \in(0,1)$.

If $\theta \neq 0$, it is clear that $r_{0}<r_{*}<1$, where $r_{*}$ is the minimal (positive) root of the equation $\rho(r)=0$ or, which is one and the same, of the equation

$$
\varphi(r):=r^{2}(2 \delta(\theta) \cos \theta-(1-L))-2 r \delta(\theta)+1-L=0 .
$$

Since $\varphi(0)=1-L>0$ and $\varphi(1)=2 \delta(\theta)(\cos \theta-1)<0$ whenever $\theta \neq 0$, we see that if

$$
2 \delta(\theta) \cos \theta \neq 1-L,
$$

then the unique root of equation (46) in the interval $(0,1)$ is

$$
\begin{aligned}
r_{*} & =\frac{\delta(\theta)-\sqrt{\delta^{2}(\theta)+[(1-L)-2 \delta(\theta) \cos \theta](1-L)}}{2 \delta(\theta) \cos \theta-1+L} \\
& =\frac{\delta(\theta)-\sqrt{[\delta(\theta)-(1-L)]^{2}+2 \delta(\theta)(1-L)(1-\cos \theta)}}{2 \delta(\theta) \cos \theta-(1-L)}, \quad \theta \neq 0,
\end{aligned}
$$

because the numerator and denominator of the last expression have the same sign.

Finally, if

$$
2 \delta(\theta) \cos \theta=1-L
$$

we see that

$$
\rho(r)=\frac{r}{1-r^{2}}(1-L-2 r \delta(\theta))
$$

and

$$
r_{*}=\frac{1-L}{2 \delta(\theta)}=\cos \theta<1
$$

whenever $\theta \neq 0$.

Proposition 26 Let $h: \mathcal{B} \rightarrow X$ be holomorphic with $h(0)=0$, let the functions $N(\theta), l(\theta)$ and $L(\theta)$ be defined by (34) and (35), and let $\rho(r)$ be defined by (45). Then for all $r \in\left(0, r_{*}\right)$, where $r_{*}$ is defined by (48) (or (51) in the case of (49)), the numbers $r$ and $\rho(r)$ are Bloch radii for the mapping $F=I-h$. Moreover, the equation $\rho^{\prime}(r)=0$ has a unique solution $r_{0} \in\left(0, r_{*}\right)$, and so the function $\rho(r)$ attains its maximum $\rho_{0}$ at this interior point $r_{0} \in\left(0, r_{*}\right) \subset(0,1)$. 
To find some explicit estimates for $r_{0}$ and $\rho_{0}$, we exploit again Proposition 6. but using another approach in order to simplify our calculations. Namely, applying Proposition 6 with $R=1$, we see that for any fixed $s \in\left(0, r_{*}\right)$,

$$
N_{s}(h)=\sup _{\|x\|=s} \operatorname{Re}\left\langle h(x), x^{*}\right\rangle \leq s^{2}[L+\mathcal{L}(\theta, s) \delta(\theta)],
$$

where $\delta(\theta)=N(\theta)-l(\theta)$, where $N(\theta), l(\theta)$ and $L$ are given by (34) and (35).

On the other hand, if we set $R=s$ in Remark 8 we get that for any $x$ such that $\|x\|=r<s$,

$$
\begin{aligned}
\operatorname{Re}\left\langle h(x), x^{*}\right\rangle & \leq r^{2} \sup _{\|u\|=1} \operatorname{Re}\left\langle h^{\prime}(0) u, u^{*}\right\rangle \cdot\left(1-\mathcal{L}_{s}(0, r)\right)+\frac{r^{2}}{s^{2}} \mathcal{L}_{s}(0, r) N_{s}(h) \\
& \leq r^{2}\left[L\left(1-\frac{2 r}{s+r}\right)+\frac{1}{s^{2}} \frac{2 r}{s+r} \cdot N_{s}(h)\right] \\
& =r^{2}\left[L \frac{s-r}{s+r}+\frac{2 r}{s+r} \cdot \frac{N_{s}(h)}{s^{2}}\right] \\
& \leq r^{2}\left[L \frac{s-r}{s+r}+\frac{2 r}{s+r}[L+\mathcal{L}(\theta, s) \delta(\theta)]\right]
\end{aligned}
$$

To simplify further our calculations we denote

$$
\begin{aligned}
& K(=K(\theta, s)):=L+\mathcal{L}(\theta, s) \delta(\theta)=L+Q, \\
& Q(=Q(\theta, s)):=\mathcal{L}(\theta, s) \delta(\theta)>0
\end{aligned}
$$

and set $R=s<1$.

Then (52) becomes

$$
\sup _{\|x\|=r} \operatorname{Re}\left\langle h(x), x^{*}\right\rangle \leq r^{2}\left[\frac{s-r}{s+r} L+\frac{2 r}{s+r} K\right]=\frac{r^{2}}{s+r}[(s-r) L+2 r K] \text {. }
$$

Now we again consider inequality (39) for $r \in(0, s]$, taking into account (53). We then obtain

$$
\sup _{\|x\|=r} \operatorname{Re}\left\langle G(x, z), x^{*}\right\rangle \leq\|z\| \cdot r-r^{2}+\frac{r^{2}}{s+r}[(s-r) L+2 r K]<0
$$

whenever

$$
\|z\|<\rho_{s}(r)=\frac{r^{2}(1+L-2 K)+r s(1-L)}{s+r}=\frac{A r^{2}+B r}{s+r},
$$

where

$$
A(=A(s))=1+L-2 K=1+L-2(L+Q)=1-L-2 Q
$$

and

$$
B(=B(s))=s(1-L)
$$


It is important to observe that for each fixed $s \in\left(0, r_{*}\right)$, the following relations hold:

(i) $\rho(s) \geq \rho_{s}(r)$,

while

(ii) $\rho(s)=\rho_{s}(s)$.

However, the investigation of the function $\rho_{s}(r), r \in(0, s]$, in order to find its maximum value can be done explicitly via quadratures.

Consider the function

$$
A(s)=1-L-2 Q(s)=\frac{(4 \delta(\theta) \cos \theta-(1-L)) s^{2}-4 \delta(\theta) s+(1-L)}{1-s^{2}} .
$$

Note that $A(0)=1-L>0$, while

$$
\begin{aligned}
A\left(r_{*}\right) & =\frac{(1-L)\left(1-r_{*}^{2}\right)-4 \delta(\theta) r_{*}\left(1-r_{*} \cos \theta\right)}{1-r_{*}^{2}} \\
& =\frac{(1-L)\left(1-r_{*}^{2}\right)-2 \delta(\theta) r_{*}\left(1-r_{*} \cos \theta\right)}{1-r_{*}^{2}}-\frac{2 \delta(\theta) r_{*}\left(1-r_{*} \cos \theta\right)}{1-r_{*}^{2}} \\
& =\frac{\rho\left(r_{*}\right)}{r_{*}}-\frac{2 \delta(\theta) r_{*}\left(1-r_{*} \cos \theta\right)}{1-r_{*}^{2}}=-\frac{2 \delta(\theta) r_{*}\left(1-r_{*} \cos \theta\right)}{1-r_{*}^{2}}<0,
\end{aligned}
$$

where $r_{*}$ is the unique positive root of the equation $\rho(r)=0$ in $(0,1)$ defined by (48). So the minimal positive root

$$
s_{*}:=\frac{2 \delta(\theta)-\sqrt{4 \delta(\theta)^{2}-(4 \delta(\theta) \cos \theta-(1-L))(1-L)}}{4 \delta(\theta) \cos \theta-(1-L)}
$$

of the equation $A(s)=0$ belongs to $\left(0, r_{*}\right)$. Moreover, for $s \in\left(0, s_{*}\right), A(s)>0$ or, which is one and the same, $Q<\frac{1-L}{2}$, and for $s \in\left(s_{*}, r_{*}\right), A(s)<0$ $\left(Q>\frac{1-L}{2}\right)$.

As we have mentioned above, for all $s \in(0,1)$, we have $\rho(s)=\rho_{s}(s)$. In particular, $\rho\left(s_{*}\right)=\rho_{s_{*}}\left(s_{*}\right)=\frac{B\left(s_{*}\right)}{2}=\frac{s_{*}(1-L)}{2}>0$.

Since $A\left(s_{*}\right)=0$, the inequality

$$
\sup _{\|x\|=r} \operatorname{Re}\left\langle G(x, z), x^{*}\right\rangle<0
$$

holds whenever

$$
\|z\|<\rho_{s_{*}}(r)=\frac{s_{*}(1-L) r}{s_{*}+r}, \quad\|x\|=r \in\left(0, s_{*}\right] .
$$

Note that in this case, $\varepsilon(r)=\frac{r}{\rho(r)}=\frac{s_{*}+r}{B\left(s_{*}\right)}$ is an affine function.

Since the derivative

$$
\rho_{s_{*}}^{\prime}(r)=\frac{2 s_{*}^{2} Q}{\left(s_{*}+r\right)^{2}}>0 \quad(Q>0),
$$


the increasing function $\rho_{s_{*}}(r), r \in\left[0, s_{*}\right]$, attains its maximum on $\left[0, s_{*}\right]$ at the point $s_{*}$, that is,

$$
\max _{r \in\left[0, s_{*}\right]} \rho_{s_{*}}(r)=\rho_{s_{*}}\left(s_{*}\right)=\rho\left(s_{*}\right)=\frac{(1-L) s_{*}}{2},
$$

and the pair $\left(s_{*}, \frac{(1-L) s_{*}}{2}\right)$ constitutes Bloch radii for $F$, with $\varepsilon\left(s_{*}\right)=\frac{2}{1-L}$.

Now we fix $s \in\left(0, s_{*}\right)$. In this case, inequality (157) holds whenever

$$
\|z\|<\rho_{s}(r)=\frac{A(s) r^{2}+B(s) r}{s+r}, \quad r \in(0, s) .
$$

The derivative

$$
\frac{d \rho_{s}(r)}{d r}=\frac{A(s)(s+r)^{2}+2 s^{2} Q}{(s+r)^{2}}>0, \quad r \in(0, s),
$$

because $A(s)>0$ for $s \in\left(0, s_{*}\right)$. Hence, $\rho$ is increasing on $[0, s]$, attains its maximum on $[0, s]$ at the point $s$, that is,

$$
\max _{r \in[0, s]} \rho_{s}(r)=\rho_{s}(s)=\rho(s)=(1-L) s-\frac{2 \delta(\theta) s^{2}(1-s \cos \theta)}{1-s^{2}},
$$

and for each $s \in\left(0, s_{*}\right)$, the pair $\left(s,(1-L) s-\frac{2 \delta(\theta)(1-s \cos \theta)}{1-s^{2}}\right)$ constitutes Bloch radii for $F$.

Next we fix $s \in\left(s_{*}, r_{*}\right)$ and let

$$
\|z\|<\rho_{s}(r)=\frac{A(s) r^{2}+B(s) r}{s+r}, \quad r \in(0, s) .
$$

In this case, $A(s)<0$ and so the equation

$$
\frac{d \rho_{s}(r)}{d r}=\frac{A(s)(s+r)^{2}+2 s^{2} Q}{(s+r)^{2}}=0
$$

or, which is one and the same,

$$
(s+r)^{2}=-\frac{2 s^{2} Q}{A(s)},
$$

makes sense. It can be seen that its minimal positive solution

$$
r^{0}=\left(\sqrt{\frac{2 Q}{2 Q-(1-L)}}-1\right) s
$$

belongs to $(0, s)$ if and only if $Q>\frac{2}{3}(1-L)$. Since $\rho_{s}(0)=0,\left.\frac{d \rho_{s}(r)}{d r}\right|_{r=0^{+}}=$ $1-L>0$ and $\frac{d^{2} \rho_{s}(r)}{d r^{2}}=-\frac{4 s^{2} Q}{(s+r)^{3}}<0$ in $(0, s)$, the function $\rho_{s}(r)$ attains its 
maximum on $[0, s]$ at the point $r^{0}$, is positive and increasing on $\left[0, r^{0}\right]$, and the pair $\left(r^{0}, \rho_{s}\left(r^{0}\right)\right)$ constitutes Bloch radii for $F$.

If $Q \leq \frac{2}{3}(1-L), \rho_{s}^{\prime}(r)$ does not vanish in $(0, s)$, and since $\rho_{s}^{\prime}(0)=1-L>0$, $\rho_{s}$ is increasing on $[0, s]$ and attains its maximum on $[0, s]$ at the point $s$, that is,

$$
\max _{r \in[0, s]} \rho_{s}(r)=\rho_{s}(s)=\rho(s)=s(1-L-Q) \geq \frac{s}{3}(1-L)>0,
$$

and the pair $(s, s(1-L-Q(s)))$ constitutes Bloch radii for $F$.

Now we summarize our conclusions in the following assertion.

Proposition 27 Let $F=I-h$, where $h \in \operatorname{Hol}(\mathcal{B}, X)$ with $h(0)=0$, the functions $N(\theta), l(\theta)$ and $L(\theta)$ be defined by (34) and (35), and let $L=L(0)<1$. Then for each $s \in\left(0, r_{*}\right)$, where $r_{*}$ is defined by (48) (or (51) in the case of (49)), the function $\rho_{s}(r)$ defined by (54) is positive on the interval $\left(0, r_{*}\right)$ and satisfies the conditions $\rho(s) \geq \rho_{s}(r)$ and $\rho(s)=\rho_{s}(s)$. Hence the pair $\left(r, \rho_{s}(r)\right)$ constitutes Bloch radii for $F$. Moreover, the following assertions hold.

(a) if

$$
s_{*}:=\frac{2 \delta(\theta)-\sqrt{4 \delta(\theta)^{2}-(4 \delta(\theta) \cos \theta-(1-L))(1-L)}}{4 \delta(\theta) \cos \theta-(1-L)},
$$

then $\varepsilon(r)=\frac{r}{\rho_{s_{*}}(r)}$ is an affine function, namely, $\varepsilon(r)=\frac{s_{*}+r}{s_{*}(1-L)}$;

(b) if $s \in\left(0, s_{*}\right)$, then $\rho_{s}(r), r \in(0, s]$, is strictly increasing and hence,

$$
\max _{r \in(0, s]} \rho_{s}(r)=\rho_{s}(s)=\rho(s)=(1-L) s-\frac{2 \delta(\theta) s^{2}(1-s \cos \theta)}{1-s^{2}} ;
$$

(c) if $s \in\left(s_{*}, r_{*}\right)$, then

$$
\begin{aligned}
& \max _{r \in\left(s_{*}, s\right]} \rho_{s}(r)=\left\{\begin{array}{ll}
\rho_{s}\left(r^{0}\right), & Q>\frac{2}{3}(1-L) \\
\rho(s), & Q \leq \frac{2}{3}(1-L)
\end{array},\right. \\
& \text { where } r^{0}=\left(\sqrt{\frac{2 Q}{2 Q-(1-L)}}-1\right) s .
\end{aligned}
$$

Note that the estimate $\frac{(1-L) s_{*}}{2} \leq \rho_{0}$ (where $\rho_{0}$ is the Bloch radius given by Proposition (26) is sharp as the following example shows.

Example 28 For $\theta=\frac{\pi}{3}, L=0$ and $\delta(\theta)=1$, we have $\rho(r)=\frac{r(1-2 r)}{1-r^{2}}$ and so $r_{*}=\frac{1}{2}$.

Then

$$
\rho_{s}(r)=\frac{A r^{2}+B r}{s+r},
$$


where $A=1-2 Q$ with $Q=\frac{s(2-s)}{1-s^{2}}$. Hence $A=\frac{s^{2}-4 s+1}{1-s^{2}}$ and the unique zero of $A$ in $(0,1)$ is $s_{*}=2-\sqrt{3}<r_{*}=\frac{1}{2}$.

For $0<s<s_{*}, A(s)>0\left(0<Q<\frac{1}{2}\right)$ and, in this case,

$$
\rho_{s}^{\prime}(r)=\frac{A(s+r)^{2}+2 s^{2} Q}{(s+r)^{2}} \geq 0 .
$$

Consequently, the function $\rho_{s}$ is increasing on $(0, s)$ and

$$
\max _{r \in[0, s]} \rho_{s}(r)=\rho_{s}(s)=\rho(s)=\frac{s(1-2 s)}{1-s^{2}} .
$$

Furthermore,

$$
\rho^{\prime}(r)=\frac{(r-2)^{2}-3}{\left(1-r^{2}\right)^{2}}
$$

and so the unique maximum of $\rho$ in $(0,1)$ is achieved at $r_{0}=2-\sqrt{3}$ and equals

$$
\rho(2-\sqrt{3})=\frac{2-\sqrt{3}}{2} .
$$

On the other hand, it is easy to calculate that $s_{*}=2-\sqrt{3}$ and $\rho_{s_{*}}\left(s_{*}\right)=\frac{2-\sqrt{3}}{2}=$ $\rho(2-\sqrt{3})$.

Remark 29 If $\theta=0$ the factor $e^{i \theta}-\mathcal{L}(\theta, r)=1-\frac{2 r}{1+r}=\frac{1-r}{1+r}$ in (40) is real and this estimate can, in fact, be replaced with a sharper one than 40), namely,

$$
\sup _{\|x\|=r} \operatorname{Re}\left\langle h(x), x^{*}\right\rangle \leq r^{2}\left[\frac{1-r}{1+r} L+N \frac{2 r}{1+r}\right],
$$

so that we do not need in this case the value

$$
l(0)=\inf _{\|x\|=1} \operatorname{Re}\left\langle h^{\prime}(0) x, x^{*}\right\rangle \leq L .
$$

In its turn formula (45) becomes

$$
\begin{aligned}
\rho(r) & =\frac{r}{1-r^{2}}\left((1-L)\left(1-r^{2}\right)-\delta(\theta) 2 r(1-r)\right)= \\
& =\frac{r}{1+r}((1-L)(1+r)-2 \delta(\theta) r) .
\end{aligned}
$$

In this situation $\rho(0)=0, \rho(1)=1-L-\delta(\theta)=1-N$ and $\rho^{\prime}(0)=1-L>0$.

So, $\rho(r)$ vanishes in $(0,1)$ if and only if

$$
N>1 \text {. }
$$

Otherwise, if

$$
N \leq 1
$$


then $\rho(r) \geq 0$ for all $r \in(0,1)$.

In particular, if $N \leq \frac{1}{2}(1+L)<1$, then also $\rho^{\prime}(r)>0$ for all $r \in(0,1)$ and

$$
\max _{r \in[0,1]} \rho(r)=\rho(1)=1-N>0 .
$$

Finally, if $N=1$, then

$$
\rho(r)=\frac{r}{1+r}((1-L)(1+r)-2(1-L) r)=\frac{r(1-r)}{1+r}(1-L)
$$

and

$$
\max _{r \in[0,1]} \rho(r)=\rho(\sqrt{2}-1)=(\sqrt{2}-1)^{2}(1-L) .
$$

In general, setting in this case $s=1$, we arrive at the following assertion.

Proposition 30 Let $h$ be a holomorphic mapping on the unit ball $\mathcal{B}$ of $X$ with $h(0)=0$ and

$$
L=\sup _{\|x\|=1} \operatorname{Re}\left\langle h^{\prime}(0) x, x^{*}\right\rangle<1 .
$$

If

$$
N=\sup _{\|x\|<R} \operatorname{Re}\left\langle h(x), x^{*}\right\rangle
$$

then

(i) $\quad N \geq L$;

(ii) Bloch radii $r_{0}$ and $\rho_{0}$ for $h$ can be given by

$$
r_{0}=\left\{\begin{array}{c}
\sqrt{\frac{2(L-N)}{1+L-2 N}}-1, \text { if } N \geq \frac{2+L}{3}, \\
1, \text { otherwise }
\end{array}\right.
$$

and

$$
\rho_{0}=\left\{\begin{array}{l}
\rho\left(r_{0}\right), \text { if } N \geq \frac{2+L}{3} \\
1-N, \text { otherwise. }
\end{array}\right.
$$

Remark 31 If, in particular, $h^{\prime}(0)=0$, then Proposition 30 coincides with Theorem 7 in [14]. Note, however, that the estimates in that theorem are still true when $h^{\prime}(0) \neq 0$, but $L=\sup _{\|x\|=1} \operatorname{Re}\left\langle h^{\prime}(0) x, x^{*}\right\rangle=0$.

\section{$5 \quad$ Radii of starlikeness and spirallikeness}

Definition 32 Let $\mu$ be a complex number with $\operatorname{Re} \mu>0$. For a domain $\mathcal{D}$ in $X$, where $0 \in \mathcal{D}$, a locally biholomorphic mapping $f: \mathcal{D} \rightarrow X$, where $f(0)=0$, is said to be $\mu$-spirallike on $\mathcal{D}$ if for each $y \in f(\mathcal{D})$ and $t \geq 0$, the curve $\exp \{-\mu t\} y$ is contained in $f(\mathcal{D})$. If, in particular, $\mu$ is a positive real number, then $f$ is said to be starlike on $\mathcal{D}$. 
Below we discuss the following two problems.

1. Let $\mu \in \mathbb{C}$ with $\operatorname{Re} \mu>0$ and $\arg \mu \in\left(0, \frac{\pi}{2}\right)$ be given. Let $\mathcal{B}$ be the open unit ball in $X$ and let $f: \mathcal{B} \rightarrow X$, where $f(0)=0$, be a $\mu$-spirallike mapping on $\mathcal{B}$. Find $r \in(0,1)$ (depending on $\mu$ ) such that $f$ is starlike on the ball $\mathcal{B}_{r}$.

2. Conversely, let $f: \mathcal{B} \rightarrow X$, where $f(0)=0$, be a starlike mapping on $\mathcal{B}$. Given $\mu \in \mathbb{C}$ with $\operatorname{Re} \mu>0$ and $\arg \mu \in\left(-\frac{\pi}{2}, \frac{\pi}{2}\right)$, find $r \in(0,1)$ such that $f$ is $\mu$-spirallike on $\mathcal{B}_{r}$.

To solve these two problems we first observe that a locally biholomorphic mapping $f: \mathcal{D} \rightarrow X$, where $f(0)=0$, is $\mu$-spirallike on $\mathcal{D}$ if and only if it satisfies the following differential equation:

$$
\mu f(x)=f^{\prime}(x) h(x),
$$

where $h: \mathcal{D} \rightarrow X$ is a semi-complete vector field on $\mathcal{D}$ (see, for example, [20]).

Since $f(0)=0$ and $f^{\prime}(0)$ is an invertible linear operator, we get that

$$
h(0)=0 \text { and } h^{\prime}(0)=\mu I .
$$

Also, it is known that if $\mathcal{D}$ is a convex domain in $X$, then the set of holomorphic semi-complete vector fields is a real cone. Therefore, in this case we can set without loss of generality $|\mu|=1$. Thus, setting $\theta=\arg \mu(|\mu|=1)$, we can reformulate our problem as follows.

Let $f: \mathcal{B} \rightarrow X$ be a locally biholomorphic mapping on $\mathcal{B}$ such that $f(0)=0$ which satisfies equation (61) with $|\mu|=1, \theta=\arg \mu \in\left(0, \frac{\pi}{2}\right)$, and let $h: \mathcal{B} \rightarrow X$ satisfy

$$
\operatorname{Re}\left\langle h(x), x^{*}\right\rangle \geq 0, x \in \mathcal{B}, x^{*} \in J(x) .
$$

Find $r \in(0,1)$ (depending on $\theta$ ) and a mapping $h_{1}: \mathcal{B}_{r} \rightarrow X$ with

$$
\operatorname{Re}\left\langle h_{1}(x), x^{*}\right\rangle \geq 0, x \in \mathcal{B}_{r}, x^{*} \in J(x),
$$

such that $f$ also satisfies the equation

$$
f(x)=f^{\prime}(x) h_{1}(x)
$$

whenever $x \in \mathcal{B}_{r}$.

It is clear that due to the uniqueness property of holomorphic solutions of differential equations, the mapping $h_{1}$ must equal $e^{-i \theta} h$, whence we have that for all $x \in \mathcal{B}$ and $x^{*} \in J(x)$,

$$
\operatorname{Re}\left\langle e^{i \theta} h_{1}(x), x^{*}\right\rangle \geq 0 .
$$

Thus to solve our problem we can use the estimates obtained in Proposition 9

Indeed, by Proposition 9, replacing $h$ by $h_{1}$ and setting $R=1$ on the lefthand side of (13), for all $x \in \mathcal{B}$ such that $\|x\|=r<1$ it holds

$$
\operatorname{Re}\left\langle h_{1}(x), x^{*}\right\rangle \geq r^{2}\left(l_{1}(0)+\mathcal{L}_{1}(\theta)\left(m_{1}(1, \theta)-L_{1}(\theta)\right)\right),
$$

where

$$
l_{1}(0)=\inf _{\|u\|=1} \operatorname{Re}\left\langle h_{1}^{\prime}(0) u, u^{*}\right\rangle=1
$$


because by (62), $h_{1}^{\prime}(0)=e^{-i \theta} h^{\prime}(0)=I$. Moreover, by (63),

$$
\begin{gathered}
m_{1}(r, \theta)=\inf _{\|x\|=r} \operatorname{Re}\left\langle e^{i \theta} h_{1}(x), x^{*}\right\rangle=\inf _{\|x\|=r} \operatorname{Re}\left\langle h(x), x^{*}\right\rangle \geq 0 . \\
L_{1}(\theta)=\sup _{\|u\|=1} \operatorname{Re}\left\langle e^{i \theta} h_{1}^{\prime}(0) u, u^{*}\right\rangle=\cos \theta
\end{gathered}
$$

and

$$
\mathcal{L}(\theta)=\frac{2 r(1-r \cos \theta)}{1-r^{2}} .
$$

So, inequality (65) holds as soon as

$$
\operatorname{Re}\left\langle h_{1}(x), x^{*}\right\rangle \geq r^{2}\left(1-\frac{2 r(1-r \cos \theta)}{1-r^{2}} \cos \theta\right):=r^{2} \varphi(r, \theta)
$$

for $x \in \mathcal{B},\|x\|=r<1, x^{*} \in J(x)$ and $0<\theta<\frac{\pi}{2}$.

Now calculations show that $\varphi(r, \theta) \geq 0$ if and only if

$$
r \leq r^{*}(\theta)=\left[\sqrt{2} \cos \left(\theta-\frac{\pi}{4}\right)\right]^{-1}<1,
$$

whenever $|\theta|<\frac{\pi}{2}$.

Thus we have proven the following result.

Theorem 33 Let $\mathcal{B}$ be the open unit ball in $X$ and let $f: \mathcal{B} \rightarrow X, f(0)=0$, be a $\mu$-spirallike mapping on $\mathcal{B}$ with $\mu=e^{i \theta},-\frac{\pi}{2}<\theta<\frac{\pi}{2}$. Then $f$ is starlike on the ball $\mathcal{B}_{r}$ for each $r \leq r^{*}(\theta)=\left[\sqrt{2} \cos \left(|\theta|-\frac{\pi}{4}\right)\right]^{-1}<1$.

Remark 34 The classical one-dimensional result of Grunsky (see, for example, [9]) asserts that any univalent function on the open unit disc is starlike on the disc centered at the origin with radius $r_{*}=\tanh \frac{\pi}{4} \simeq 0.65$. It is clear that in the special case of spirallike functions we have obtained a better estimate since $\min r^{*}(\theta)=r^{*}\left(\frac{\pi}{4}\right)=\frac{1}{\sqrt{2}} \simeq 0.71>r_{*}$. In the one-dimensional case this estimate was obtained by Robertson [22].

To solve the second problem described above we just use Theorem 23 and Remark 24 to obtain the following result.

Theorem 35 Let $\mathcal{B}$ be the open unit ball in $X$ and let $f: \mathcal{B} \rightarrow X, f(0)=0$, be a starlike mapping on $\mathcal{B}$. Then for each $-\frac{\pi}{2}<\theta<\frac{\pi}{2}$ and for each $0<$ $r \leq r(\theta)=\frac{1-|\sin \theta|}{\cos \theta}<1$, the mapping $f$ is $\mu$-spirallike on the ball $\mathcal{B}_{r}$ with $\mu=e^{i \theta}$.

The following example suggested by one of the referees shows that the estimates in Theorems 33 and 35 are sharp. 
Example 36 Let $u \in X$ be a unit vector and let $M$ be a subspace of $X$ such that each $x \in X$ has a unique representation $x=x_{1} u+\widehat{x}$, where $x_{1} \in \mathbb{C}$ and $\widehat{x} \in M$. Consider the mappings $f_{\theta}: \mathcal{B} \rightarrow X$, defined by $f_{\theta}(x)=\frac{1}{\left(1-x_{1}\right)^{1+\exp (2 i \theta)}} x$, $\theta \in\left[0, \frac{\pi}{2}\right)$. It can be seen that $f_{0}$ is starlike on $\mathcal{B}$ while $f_{\theta}, \theta \in\left(0, \frac{\pi}{2}\right)$, is $\mu$ spirallike on $\mathcal{B}$ with $\mu=e^{i \theta}$. Calculations show that for these mappings the estimates given in Theorems 33 and [35] cannot be improved.

Acknowledgments. We are very grateful to the anonymous referees for their many useful comments and helpful suggestions.

\section{References}

[1] D. Aharonov, S. Reich, D. Shoikhet, Flow invariance conditions for holomorphic mappings in Banach spaces, Math. Proc. R. Ir. Acad. 99A, 93-104 (1999).

[2] F. Bracci, M. Elin, D. Shoikhet, Growth estimates for pseudo-dissipative holomorphic maps in Banach spaces, J. Nonlinear Convex Anal. 15, 191198 (2014).

[3] F. Bracci, Y. Kozitsky, D. Shoikhet, Abel averages and holomorphically pseudo-contractive maps in Banach spaces, J. Math. Anal. Appl. 423, 1580-1593 (2015).

[4] R. B. Burckel, An Introduction to Classical Complex Analysis, Vol. 1, Academic Press, New York and London, 1979.

[5] S. B. Chae, Holomorphy and Calculus in Normed Spaces, Marcel Dekker, New York, 1985.

[6] C. J. Earle, R. S. Hamilton, A fixed point theorem for holomorphic mappings, Proc. Symp. Pure Math., Vol. 16, Amer. Math. Soc., Providence, RI, 61-65 (1970).

[7] M. Elin, S. Reich, D. Shoikhet, Holomorphically accretive mappings and spiral-shaped functions of proper contractions, Nonlinear Anal. Forum 5, 149-161 (2000).

[8] T. Franzoni, E. Vesentini, Holomorphic Maps and Invariant Distances, North-Holland, Amsterdam, 1980.

[9] G. Goluzin, Geometric Function Theory of Complex Variable, GITTL, Moscow, 1952. 
[10] I. Graham, H. Hamada, G. Kohr, Radius problems for holomorphic mappings on the unit ball in $\mathbb{C}^{n}$, Math. Nachr. 279, 1474-1490 (2006).

[11] I. Graham, H. Hamada, T. Honda, G. Kohr, K. H. Shon, Distortion and coefficient bounds for Carathéodory families in $\mathbb{C}^{n}$ and complex Banach spaces, J. Math. Anal. Appl. 416, 449-469 (2014).

[12] L. A. Harris, The numerical range of holomorphic functions in Banach spaces, Amer. J. Math. 93, 1005-1019 (1971).

[13] L. A. Harris, On the size of balls covered by analytic transformations, Monatshefte Math. 83, 9-23 (1977).

[14] L. A. Harris, S. Reich, D. Shoikhet, Dissipative holomorphic functions, Bloch radii, and the Schwarz lemma, J. Anal. Math. 82, 221-232 (2000).

[15] V. Khatskevich, S. Reich, D. Shoikhet, Global implicit function and fixed point theorems for holomorphic mappings and semigroups, Complex Variables 28, 347-356 (1996).

[16] G. Kresin, V. G. Maz'ya, Sharp Real-Part Theorems. A Unified Approach, Lecture Notes in Mathematics, 1903, Springer, Berlin, 2007.

[17] S. Reich, On fixed point theorems obtained from existence theorems for differential equations, J. Math. Anal. Appl. 54, 26-36 (1976).

[18] S. Reich, D. Shoikhet, Generation theory for semigroups of holomorphic mappings in Banach spaces, Abstr. Appl. Anal. 1, 1-44 (1996).

[19] S. Reich, D. Shoikhet, Semigroups and generators on convex domains with the hyperbolic metric, Atti Accad. Naz. Lincei Cl. Sci. Fis. Mat. Natur. Rend. Lincei 9, 231-250 (1997).

[20] S. Reich, D. Shoikhet, Nonlinear Semigroups, Fixed Points, and Geometry of Domains in Banach Spaces, Imperial College Press, London, 2005.

[21] S. Reich, D. Shoikhet, J. Zemánek, Ergodicity, numerical range, and fixed points of holomorphic mappings, J. Anal. Math. 119, 275-303 (2013).

[22] M. S. Robertson, Radii of star-likeness and close-to-convexity, Proc. Amer. Math. Soc. 16, 847-852 (1966).

Filippo Bracci, Dipartimento di Matematica, Università di Roma "Tor Vergata", Via della Ricerca Scientifica 1, 00133 Roma, Italy

E-mail address: fbracci@mat.uniroma2.it 
Marina Levenshtein, Department of Mathematics, ORT Braude College, 21982 Karmiel, Israel

E-mail address: marlev@braude.ac.il

Simeon Reich, Department of Mathematics, The Technion - Israel Institute of Technology, 32000 Haifa, Israel

E-mail address: sreich@tx.technion.ac.il

David Shoikhet, Department of Mathematics, ORT Braude College, 21982 Karmiel, Israel

E-mail address: davs@braude.ac.il 